\title{
FUZZY SET-POINT WEIGHT FOR PID CONTROLLER BASED ON ANTLION OPTIMIZER TO CONGESTION AVOIDANCE IN TCP/AQM ROUTERS
}

\author{
Haneen S. Abdulkareem ${ }^{1}$, Osama A. Awad ${ }^{2}$ \\ ${ }^{1,2}$ College of Information Engineering, Al-Nahrain University, Baghdad, Iraq \\ $\left\{\right.$ haneen.saad ${ }^{1}$, usamaawad $\left.{ }^{2}\right\} @$ coie-nahrain.edu.iq \\ Received:22/9/2019, Accepted:22/11/2019
}

\begin{abstract}
The delay in delivering packets and packets loss in computer networks is due to the problem of congestion in TCP/ AQM routers, and to solve this problem, the transmission control protocol and active queue management worked together. TCP provides secure data transfer and designed to handle congestion after its occurrence. AQM predicts congestion and tries to resolve the problem before it occurs. In this work, the Fuzzy- PID (FPID) controller is presented to manage the avoidance of congestion problems associated with TCP networks. The approach is based on hybridization between the Fuzzy Logic Controller (FLC) and the Proportional-Integral-Derivative(PID) controller, with the structure of Fuzzy Set- Point- Wight(FSW) for PID controller that optimized by Antlion Optimizer(ALO) using a linearized TCP congestion model. The target is to control the queue length of the router for the queue level in demand. The FPID controller shows good robustness for different scenario of the TCP network and the queue length response showed fast tracking capability with good robustness to network parameters changing in comparison of using PID controller. All simulations are carried out using MATLAB 2017b.
\end{abstract}

keywords: Active Queue Management (AQM), Antlion- Optimizer (ALO), TCP, PID controller, Fuzzy- PID (FPID), Fuzzy Set- point Weight (FSW).

\section{INTRODUCTION}

The network congestion takes place when the number of incoming data is bigger than the ability of the router to accommodate. This leads to massive delay and packets loss, to avoid this mess, congestion control algorithm was needed to manage the networks. In 1988 Transmission Control Protocol(TCP) was incepted. TCP has the main roll in network congestion control where it provides end-to- end congestion control by providing the sender with the congestion window(cwnd) that carry the maximum number of the unacknowledged acceptable packets, that means it can prevent congestion growth, however with rapid development in the communication world, this technique was not enough. New technique that foresees congestion before it happens is necessary needed. This technique was an Active Queue Management algorithm (AQM). It is worked together with end- to- end congestion control to decrease the packet loss and delay in the network, to enhance the Quality of Service (QoS) [1], [2].

\section{RELATED WORK}

In 2016 H. Ali [3] proposed a Multi- Wavenet PID controller (AMWPID) for congestion avoidance in TCP/ AQM routers. The comparison test with other AQM algorithms shows that the developed AMWPID outperforms the other approaches in term of the number of TCP flows, link capacity and round- trip time. Yang in 2013 [4] proposed the LQ- servo controller for TP/ AQM network where the network has time- varying capacity. The aim of the proposed controller is to avoid congestion in the network. Sen in 2018 [5] proposed hybridization between Fuzzy Set- Point weight structure and PID controller. The fuzzy rule base design based on the sliding mode technique. The proposed controller use for closed-loop 
application. Visioli [7] proposed the first time tuning set- point weight by the fuzzy logic controller and use it to control the proportional gain in PID controller. Also use Ziegler- Nichols in the PID parameter's tuning. S. Mirjalili proposed in 2015 [8] a new optimizer method that simulates hunting behavior of the Antlions in nature, where it tested for 19 problems and proved it's successes.In [9] proposed a Fuzzy- PID like- Gain Scheduling (F- PIDGS) controller, to handle the time delays in NCS depending on the online measured actual time delay in the network, by utilizing both fuzzy and gain scheduling control aspects. To investigate the performance of the F- PIDGS controller with the aid of discrete- event simulation models. Domanski in 2017 [10] examined the fractional- order- PID controller performance as a mechanism for AQM. The traffic self-similarity influence on the utilization of a TCP network is investigated. The impact of PID parameters selection and traffic self-similarity degree on the behavior of queue length were proved. A memoryless ' $\mathrm{H} \infty$ ' controller was designed in 2016 for TCP/ IP routers [11]. The controller designed to be robust for time- variant networks to keep stability and performance of network in a critical situation, using "standard linear matrix inequality techniques". The parameters of controller was optioned. Monisha [12] proposed a new controller based on hybridization between fuzzy- PID controller with Random Early Detection (RED) method to avoid congestion in Wireless Sensor Networks. In 2018 [13] presented an investigation of the performance of a PID- based congestion control solution for high- speed IP networks. It uses measurements of round- trip time and receiver delivery rate to reach and keep maximum available bottleneck performance and constant node buffer load caused by bottleneck queue on some low level. This algorithm can be effective in high-speed IP networks and delay- sensitive applications. Karthikeyan in 2014 [14] use a fuzzy set point weight(FSW) structure with Fractional- Order- PID controller(FOPID), the controller designed to enhance the response in the closed- loop parallelcascade controller. The proposed controller compared with classical PID controller and found that, the proposed controller was faster in term of settling time. In 2019, [15] proposed FOPID controller, to control the variation in the probability of dropping packet when occupancy of the buffer continuously growth. The probability function chooses its shape through parameters selection. In this paper, a control strategy based on a fuzzy set-point weight approach combined with the PID controller was suggested for congestion control. The parameters tuned using the Antlion optimization (ALO) algorithm.

\section{THE TCP BEHAVIOR MODEL}

TCP model dynamical behavior is introduced based on the stochastic differential equation and fluid flow analysis. If the mechanism of the TCP timeout is ignored then the developed state- space model is given by the following [4]:

$$
\begin{gathered}
W^{\prime}(t)=\frac{1}{R(t)}-\frac{W(t) W(t-R(t))}{2 R(t-R(t))} p(t-R(t)) \\
q^{\prime}(t)=\frac{W(t)}{R(t)} N(t)-C
\end{gathered}
$$

Where $W^{\prime}(t)$ represents the derivative of ' $\mathrm{w}(\mathrm{t})^{\prime}, q^{\prime}(t)$ represents the ' $\mathrm{q}(\mathrm{t})$ ' derivation. the term ' $\mathrm{W}$ ' refers to the average (packets) size of TCP window, q refers to average packets of queue length, the round trip time (s) $R(t)$, ' C' links capacity (packets per second), $N(t)$ is TCP sessions representing the load factor, and ' p' probability of (drop/mark) packets, If a constant number of TCP sessions $N(t) \equiv N$ is assumed with constant round trip time $R(t) \equiv R_{0}$. Then the linearized 
TCP dynamical model for AQM routers will be:

$$
\begin{gathered}
\delta W^{\prime}(t)=-\frac{2 N}{R_{0}^{2} C} \delta W(t)-\frac{R_{0} C^{2}}{2 N^{2}} \delta p\left(t-R_{0}\right) \\
\delta q^{\prime}(t)=\frac{N}{R_{0}} \delta W(t)-\frac{1}{R_{0}} \delta q(t)
\end{gathered}
$$

\section{FSW FOR PID CONTROLLER}

The structure of the Fuzzy- PID controller designed according to the " fuzzy set- point- weight" (FSW) structure shown in Fig. 1. The FSW structure effective in reducing the overshoot without increasing in the rise time [5-7]. FSW structure dependent on the fuzzy inference system " $f(t)$ " for determining the value of the weight " $b(t)$ " that multiplies the "set- point (ysp)" for the proportional action " kp" based on the current output error and its time derivative. The use of set- point alone (without fuzzy) is possible where it is effective in reducing the overshoot. But, also has disadvantage represented in an increase in the rise time since the performance of the proportional action is slightly reduced. The value of the weight $b(t)$ determined depending on the current value of the system error, and its time derivative. The PID controller standard form in the time domain is [7] :

$$
u(t)=k_{p} e(t)+k_{d} \frac{d e(t)}{d t}+k_{i} \int_{0}^{t} e(t) d t
$$

The set- point for the proportional action weighted by means of a constant to get :

$$
u(t)=k_{p} e_{p}(t)+k_{d} \frac{d e(t)}{d t}+k_{i} \int_{0}^{t} e(t) d t
$$

Where:

$$
\begin{gathered}
e_{p}(t)=b(t) y_{s p}-y(t) \\
b(t)=w f(t)
\end{gathered}
$$

w: positive constant parameter less than or equal to 1 (An effective way to keep overshoot in law value is to weight the set- point for the proportional action by means of a constant less than 1.)

$f(t)$ : fuzzy controller output, the FSW gives 2- degree of freedom, where one part of controller dedicated for reduction disturbances of load and the second part dedicated to the set- point, finally a simple summary to completely understood of the methodology, the fuzzy controller output ' $f(t)$ ' is companied with' $w$ ' constant parameter, the output is ' $b(t)$ ', the ' b(t)' will multiply by ' ysp' set- point.

\section{Antlion Optimizer (AlO)}

In 2015 Mirjalili [8] introduced a new optimization algorithm that simulates hunting behavior of the antlion in nature called ant-lion algorithm (ALO). Let us describe the ant- lion behavior in nature, firstly ant-lion start dig " trap" in cone shape and waiting its prey, the prey " mostly ant" walking randomly around trap, when victim enter the trap, ant- lion start to throw sand toward prey for speeding up its fall, when prey fall in trap ant- lion feed on it and be ready for next victim. The antlions strategy in hunting summaries in five steps.

1) Random Walk of Ants: During Searching on food Ants moving randomly over " area" a search space, at same time antlions Waite probable chance to hunting prey. 
2) Building trap: During optimization, the antlions choosing is based on the roulette wheel based on their fitness. This strategy gives high chances to the fitter antlions for hunt prey.

3) Entrapment of ants in traps: The antlions trap that building in the search space are affected on prey when it moving, so the ant should move around trap otherwise it will fall in the trap.

4) Sliding ants towards antlion: To speed up the falling of prey, antlion throw sand toward the ant

5) Catching Preys and Rebuilding the Traps: After catching prey and eat it, the antlion make some edit on the trap. The ALO Flowchart is clarify in Fig. 2.

\section{NETWORK TOPOLOGY}

To judge the proposed controller and evaluate its performance, the case study shown in Fig. 3, will be considered. where the TCP network of AQM routers consists of 2 routers that connected through a Bottleneck link with '3750' packets/ sec bandwidth capacity (C).Each packet has 500 bytes, which means' $15 \mathrm{Mbps}$ ' link capacity, all other links in the network have the same link capacity. Round-Trip Time (RTT) $\left(R_{0}\right)$ equals to '0.218' seconds, either about the size of the queue, it will be ' 300 packets', with propagation delay '5msec', the maximum allowable queue length will be 800 packets. and on both routers, the number of a TCP flow(N) will be 60. the proposed controller is set up in Router 1, while the drop- tail set on Router 2.

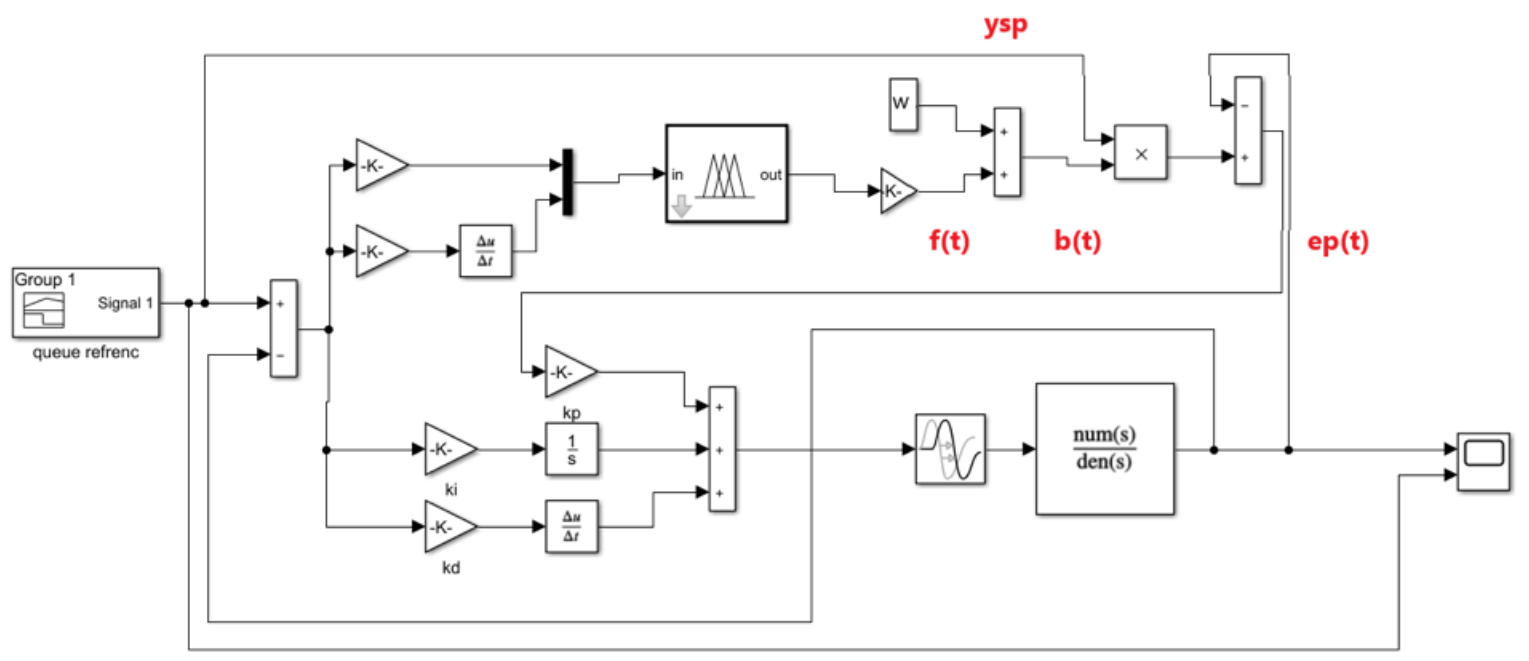

Figure 1: FSW for PID controller 




Figure 2: ALO flowchart

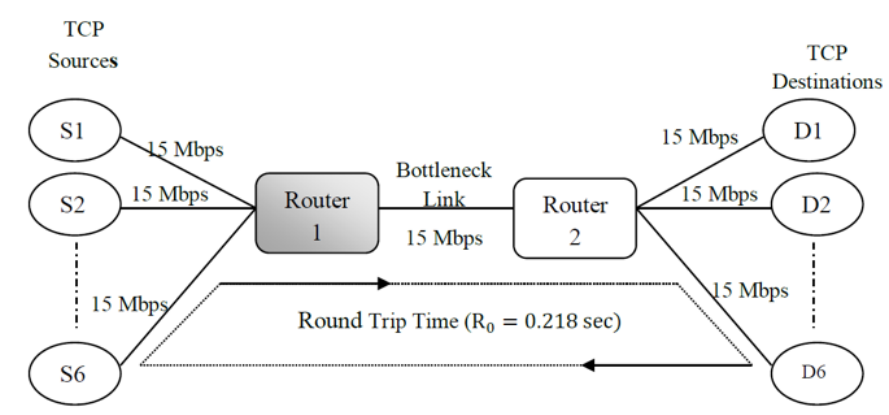

Figure 3: Network topology case study 


\section{WORK METHODOLOGY}

The problem of the network congestion model is that it is not fixed, because it is related to the network load which is varying all time. It mainly depends on the load factor and capacity of transmission link. so, there is no alternative to using dynamic controller in such systems, to check the performance of proposed controller, it must be compared with conventional controller such PID controller.

1) PID Controller: PID controller was built using Eq. 5, the PID parameters $\left(k_{p}, k_{i}, k_{d}\right)$ were optimized by the Antlion optimizer.

2) FPID controller: The structure of the FPID controller designed according to FSW structure, either about Fuzzy Logic Controller, Triangular membership functions are used for input variables as it is shown in Fig. 4, For the output variable u, singleton membership functions are defined as in Fig. 5a, The fuzzy PID controller rule base composed of $25(5 \times 5)$ rules Fig. 5 b.

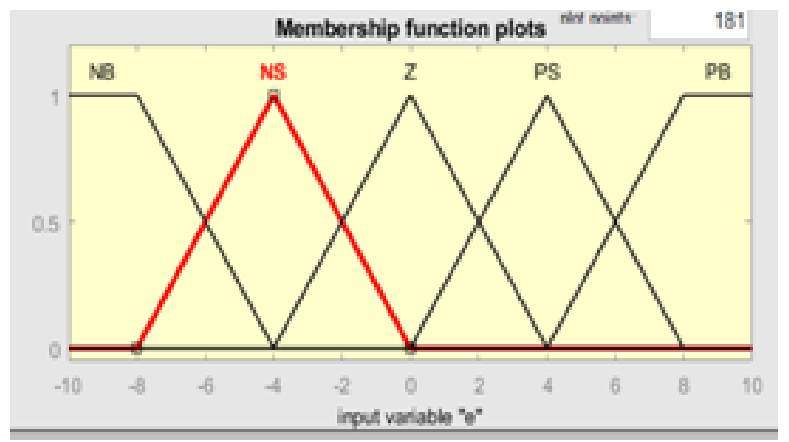

(a) Error membership function

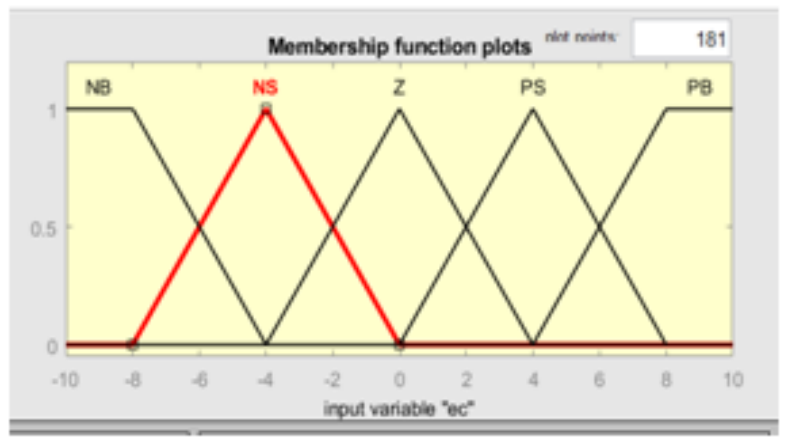

(b) Error change membership function

Figure 4: Error and error change membership function

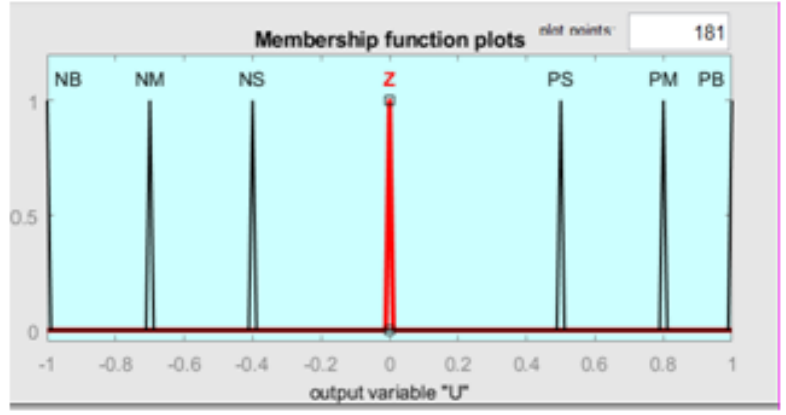

(a) The output membership function

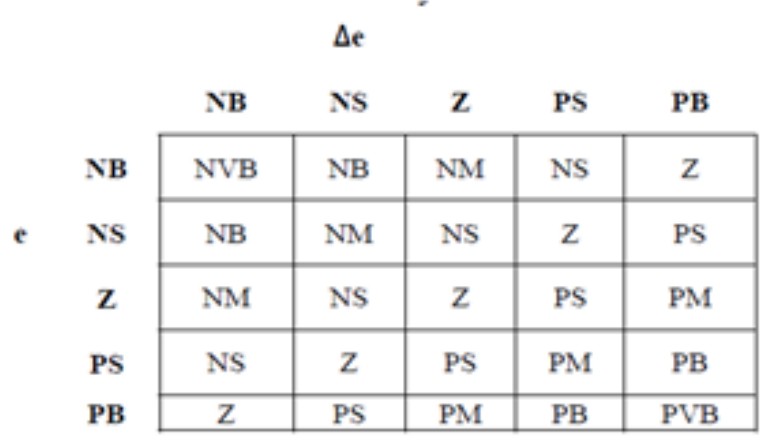

(b) Fuzzy rule base

Figure 5: The output membership function and fuzzy rule base 


\section{Simulation Results}

The TCP/ AQM model of network parameters in (Fig. 3) was considered, the queue length size (reference input) assumed to be of a rectangular form that periodically changes every $50 \mathrm{~s}$, " Eq. 8 ". the simulation starts on the system without using any controller, the system response is shown in Fig. 6.

$$
q_{\text {ref }}=\left\{\begin{array}{cc}
300 & 0<t<50 s \\
200 & 50<t<100 s \\
400 & 100<t<150 s \\
200 & 150<t<200 s
\end{array}\right.
$$



Figure 6: System response without controller

This figure shows clearly the failure of system in tracking the queue length of input and keep it with desired threshold. This leads to high number of packets drops and a large queue delay, and this, in turn, causes network instability. to enhance the utilization of network, (PID, FPID) controllers are designed to achieve a better performance of queue track, with $k_{p}=8.96 e-06, k i=3.82 e-06, k_{d}=2.39 e-06$ choosing by ALO and $\mathrm{w}=0.5$.

\section{1) Test 1}

For the standard case study of the network shown in Fig. 3, with $\mathrm{N}=60$ and $\mathrm{C}=15 \mathrm{Mbps}$ the response of system with (PID, FPID) controllers shown in (Fig. 7), and the response scale factor of the controllers is described in Table I, where both controllers can track the desired queue length.

\section{2) Test 2}

As known the value of RTT (packet trip from' source host' to ' destination', back to source in seconds) is not constant, where it is continuously changing, to study the effect of decreasing RTT on queue length and behavior of the proposed controller in such situation. The value of RTT is decreased by $60 \%$ to increase congestion where $\mathrm{R}=$ $0.088 \mathrm{~s}$, while values of $\mathrm{N}$ and $\mathrm{C}$ are kept unchanged. 
TABLE I

Dynamic Performance $c / c s$ of The Two Controllers for N=60, C=15MbPS

\begin{tabular}{|c|c|c|c|}
\hline $\mathrm{N}=60, \mathrm{C}=15$ & PID & FPID & Enhancement rate(PID-FPID) \\
\hline Rise time (s) & 2.5564 & 1.4859 & $44 \%$ \\
\hline Settling time (s) & 7.0177 & 4.9399 & $30 \%$ \\
\hline Overshoot $\%$ packets & 0 & 2.4712 & - \\
\hline ISE & $9.233 \mathrm{e}-06$ & $1.0107 \mathrm{e}-07$ & $98.8 \%$ \\
\hline
\end{tabular}

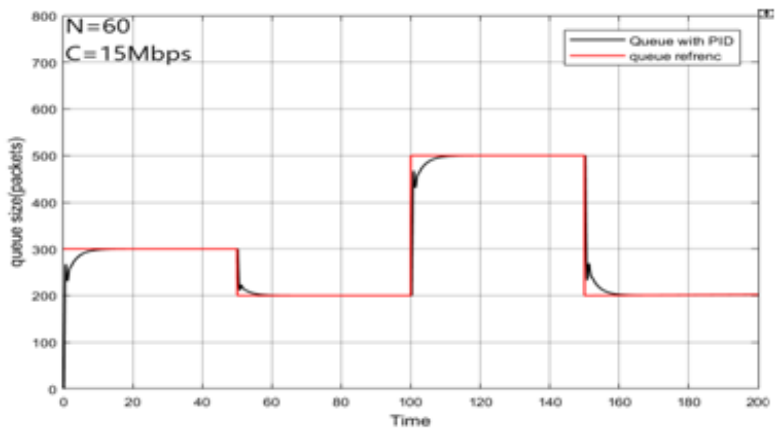

(a) PID Controller

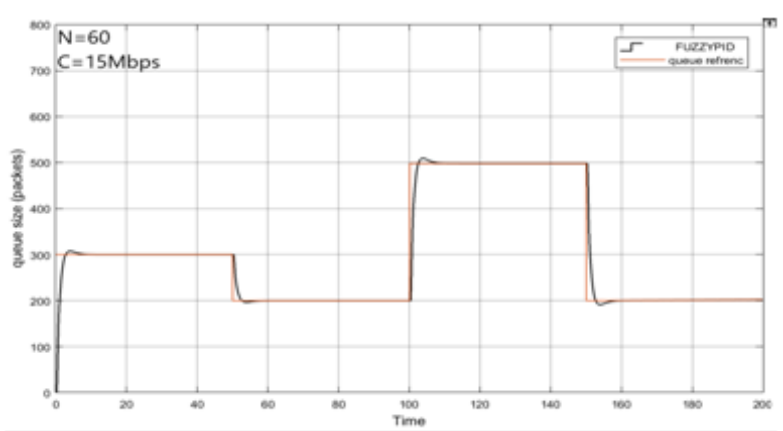

(b) FPID controller

Figure 7: The Output queue length of network with $\mathrm{N}=60$ and $\mathrm{C}=15 \mathrm{Mbps}$ for

(a) PID Controller

(b) FPID Controller

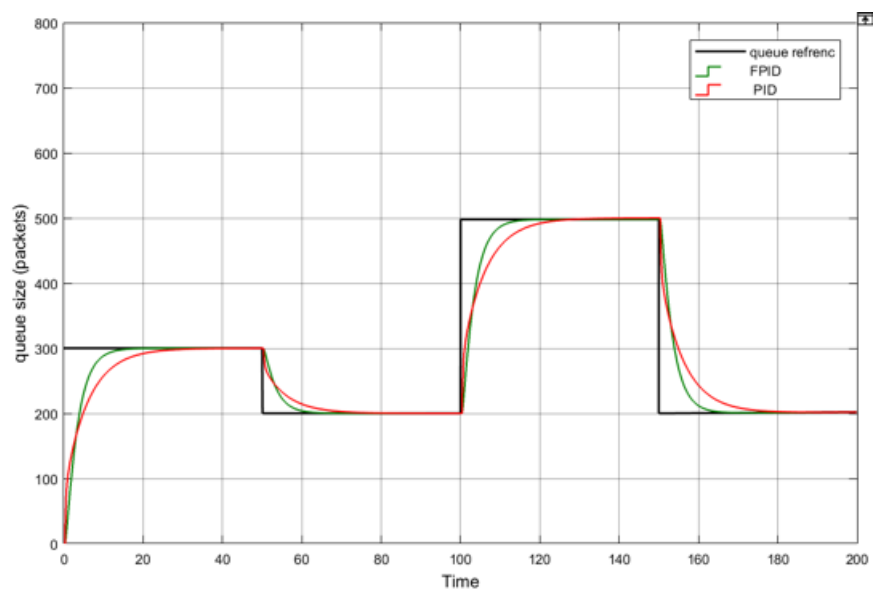

Figure 8: The Output queue length of (PID, FPID) controllers when, N=60 and $\mathrm{C}=15 \mathrm{Mbps}, \mathrm{R}=0.088 \mathrm{~s}$

TABLE II

DyNAMiC PERFormance $c / c s$ OF THE Two Controllers FOR N=60, C=15MbPS

\begin{tabular}{|c|c|c|c|}
\hline $\mathrm{N}=60, \mathrm{C}=15, \mathrm{R}=0.008$ & PID & FPID & Enhancement rate(PID-FPID) \\
\hline Rise time (s) & 11.7338 & 6.5158 & $44 \%$ \\
\hline Settling time (s) & 21.7216 & 11.8115 & $45.7 \%$ \\
\hline Overshoot1\% packets & 0 & 0 & - \\
\hline ISE & 0.214 & 0.0013 & $99 \%$ \\
\hline
\end{tabular}


As clear from Fig. 8 and Table II. the rise time and settling time have continuously increased with PID controller that may leading to packets dropped and delay. The delay comes from long-duration response of long settling time and rise times. But the FPID controller tries to keep the rise time and settling time in acceptable value.

\section{3) Test 3}

To test the robustness of the proposed controller, the link capacity (C) is decreased to 7.5Mbps. The value of load factor $(\mathrm{N})$ is set to 80. Fig. 9 depicts the system response of the controllers and Table III depicts some dynamic characteristics of the system response.

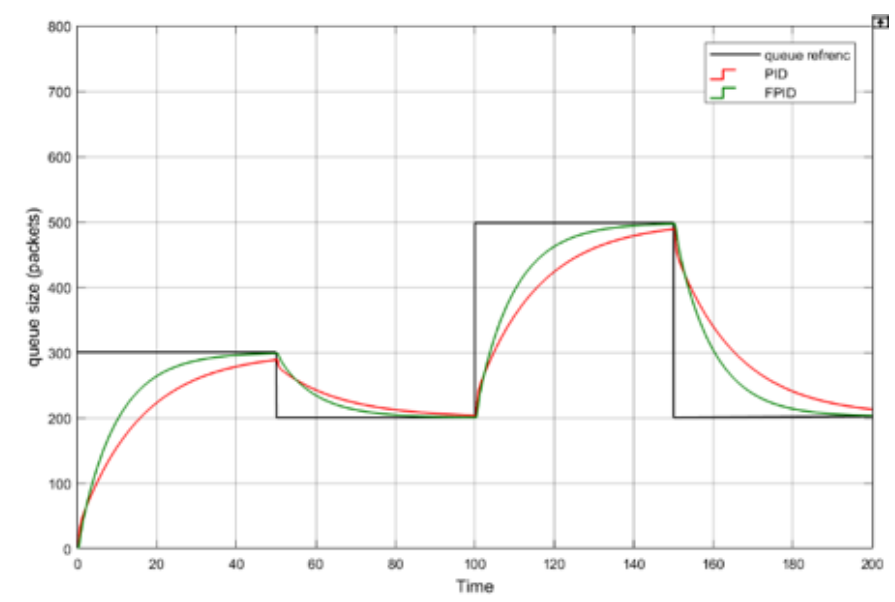

Figure 9: The Output queue length of the (PID, FPID) Controllers when, $\mathrm{N}=80$ and $\mathrm{C}=7.5 \mathrm{Mbps}$

TABLE III

Dynamic Performance $c / c s$ OF The Two Controllers for N=80, C=7.5 MbPs

\begin{tabular}{|c|c|}
\hline $\mathrm{N}=80, \mathrm{C}=7.5$ & FPID \\
\hline Rise time (s) & 20.4092 \\
\hline Settling time (s) & 35.1310 \\
\hline Overshoot \% packets & 0 \\
\hline ISE & 9.85 e-03 \\
\hline
\end{tabular}

From Fig. 9, one can see that, the PID controller was inefficient in tracking the desired queue length With more TCP flows, the system becomes unstable as most of the incoming packets will be dropped. Hence the queue delay will be very large, causing high packet loss and the network throughput will be very low and become unacceptable by the users. while the FPID controller, tries to keep the system under control. This tolerance of FPID controller regards the Fuzzy- Set- PointWeight structure.

\section{Conclusions}

In this paper, the proposed controller was able to keep queue under control in a critical situation and it is Outperform on the PID controller, in terms of Rising time, Settling time and ISE. where FPID enhances rise time to $44 \%$ and settling time to $45 \%$ to avoids the delay that comes from the long-duration response of long settling time and rise times as clear from Test 2. Furthermore, we can note from Test 3 that FPID shows a high ability to face environmental changes in network 
parameters. The FPID controller shows a very good tracking capability for the desired queue size it gives better congestion avoidance compared with PID controller and decreases the packet loss and delay leading to increase Qos. The Fuzzy set-point weight for PID and ALO optimizer help in increase controller robustness because of the two degrees of tolerance provided by FSW structure and the good parameter tuning of Antlion- optimizer. The proposed controller outperforms on the AMWPID controller mention in [3] for the same case. While achieving an approximate value of hybrid fuzzy logic controller [22]. see Table IV.

TABLE IV

Comparation of The Proposed Controller With Others Controller

\begin{tabular}{|c|c|c|c|}
\hline $\mathrm{N}=60, \mathrm{C}=15$ MBPS & PROPOSE CONTROLLER & AMWPID CONTROLLER [3] & HYBRID FUZZY LOGIC CONTROLLER [22] \\
\hline Rise time (s) & 1.4859 & 1.8 & $1.551 \%$ \\
\hline Settling time $(\mathrm{s})$ & 4.9399 & 8 & $1.733 \%$ \\
\hline Overshoot $\%$ packets & 2.4712 & 0 & $2.510 \%$ \\
\hline
\end{tabular}

\section{REFERENCES}

[1] J . Aikat, K. Jeffay, and F. D. Smith, " The Effects of Active Queue Management and Explicit Congestion Notification on Web Performance" , IEEE/ ACM Trans. Netw., no. 1, pp. 1- 18, 2005.

[2] A. K. Sharma, " A Survey on Active Queue Management Techniques" , Int. J. Eng. Comput. Sci., vol. 5, no. 11, pp. $18993-18997,2016$.

[3] Hind Ali, Osama A. Awad, " A Multi- Resolution Wavelet PID Controller for Internet AQM Routers " , Int. J. Innov. Res. Comput. Commun. Eng. ( An ISO Certif. Organ. ), vol. 3297, no. 6, pp. Vol. 4, Issue 5, May 2016.

[4] J. Yang, etc. , "LQ- servo congestion control for TCP/ AQM system in a wireless network environment" , Int. J. Control Autom. , vol. 6, no. 3, pp. 281- 290, 2013.

[5] K. Sen, B. Chakraborty, A. Gayen, and C. Dey, " Fuzzy Rule- Based Set Point Weighting for PID Controller Kausik" , Springer Singapore, vol. 537, no. January 2018.

[6] M. Suresh, R. R. Hemamalini, and G. J. Srinivasan," Fuzzy Logic Based Set- Point Weighting Controller Tuning for an Internal Model Control Based PID Controller", Sensors Transducers J. , vol. 109, no. 10, pp. 29- 42, 2009

[7] A. Visioli, " Fuzzy logic based set- point weight tuning of PID controllers", IEEE Trans. Syst. Man, Cybern. Part systems Humans. , vol. 29, no. 6, pp. 587- 592, 1999.

[8] S . Mirjalili," The ant lion optimizer" , Adv. Eng. Softw. , Elsevier Ltd. , vol. 83, pp. 80- 98, 2015.

[9] O. A. A. Isra'a. Salim1, " Gain Scheduling PID Controller For Networked Control System with Random Delay", Int. J. Enhanc. Res. Sci. Technol. Eng. , vol. 4 Issue 2, pp. 2319- 7463, 2015.

[10] A. Domanski, J. Domanska, T. Czachorski, J. Klamka, and J. Szygula, " Self- similarity Traffic and AQM Mechanism BasedonNon- integer Order PI $\alpha$ D $\beta$ Controller", Springer International Publishing AG 2017.

[11] N. El Fezazi, O. Lamrabet, F. El Haoussi, E. H. Tissir, T. Alvarez, and F. Tadeo, " Robust controller design for congestion control in TCP/ IP Routers", Conf. Syst. Control. ICSC 2016.

[12] V. Monisha and T. Ranganayaki, " A Service Differentiation Aware Dynamic Random Early Detection and Optimized Fuzzy Proportional Integral Derivative for Active Queue Management Congestion Control in Mobile Wireless Sensor Network", vol. 16, no. 7, pp. $30-40,2018$.

[13] Nikolai Mareev, Dmitry Kachan, " Efficiency of a PID-based Congestion Control for High- speed IP- networks" (ICAIIT), March 2018.

[14] R. Karthikeyan, etc. , " Fuzzy Fractional Order PID Based Parallel Cascade Control System" , Adv. Intell. Syst. Comput. , vol. 235, pp. V- VI, 2014.

[15] A. Domanski, J. Domanska, etc, " The AQM dropping packet probability function based on a non- integer order PI $\alpha \mathrm{D} \beta$ controller" , Lect. Notes Electr. Eng., vol. 496, pp. 36- 48, 2019.

[16] M. M. Abualhaj, A. A. Abu- Shareha, , " FLRED: an efficient fuzzy logic- based network congestion control method" , Neural Comput. Appl. , vol. 30, no. 3, pp. 925- 935, 2016.

[17] I. S. Jesus and R. S. Barbosa, " Application of fuzzy fractional PD+I controllers tuned by a Genetic Algorithm", IECON Proc. (Industrial Electron. Conf. , no. November 2013, pp. 3335- 3340, 2013.

[18] Ghasem Kahe , Amir Hossein Jahangir, " A self- tuning controller for queuing delay regulation in TCP/ AQM networks Ghasem" , Springer Sci. Media, LLC, part Springer Nat. 2018, 2018.

[19] S.Chebli, A.Elakkary, N.Sefiani, and N.Elalami2, " PI Stabilization for Congestion Control of AQM Routers with Tuning Parameter Optimization Article" , Int. J. Interact. Multimed. Artif. Intell. , vol. 4, no. Special Issue on Artificial Intelligence Underpinning, 2016.

[20] M. Mani, O. Bozorg- Haddad, and X. Chu, " Ant lion optimizer (ALO) algorithm" , Studies in Computational Intelligence, vol. 720. pp. 105- 116, 2018.

[21] I. Iancu, " A Mamdani Type Fuzzy Logic Controller" , in InTech, E. Dadios, Ed. IntechOpen, 2012.

[22] Z. A. Karam, " Hybrid Fuzzy Congestion Controllers for Computer Networks Tuned by Modified Particle Swarm Optimization" , Int. J. Adv. Telecommun. Electrotech. Signals Syst. , vol. 7, no. 2, p. 17, 2018.

[23] N. Mareev, D. Kachan, K. Karpov, D. Syzov, and E. S. and Y. Babich, " Efficiency of a PID- based Congestion Control for High-speed IP-networks" , Proc. 6th Int. Conf. Appl. Innov. IT, (ICAIIT), no. April, pp. 84- 90, 2018. 